\title{
Chinakräuter gegen Endometriose?
}

\section{Die traditionelle chinesische Medizin (TCM) kennt ein Kraut gegen so ziem- lich jedes Leiden. Die Wirksamkeit dieser Kräutermixturen wird (schein- bar) evidenzbasiert bestätigt.}

- Chinesische Forscher randomisierten 320 Patientinnen, die alle wegen Endometriose operiert worden waren, in zwei Gruppen. Die Experimentalgruppe erhielt drei bis sechs Monate lang chinesische Heilkräuter, die individualisiert verabreicht wurden, während die Kontrollgruppe mit herkömmlichen Medikamenten therapiert wurde. Als Hauptzielkriterium galt die Lebensqualität.

Dieser Parameter verbesserte sich in beiden Gruppen, ohne dass Gruppendifferenzen erkennbar waren. Teilbereiche der Lebensqualität zeigten posi- tive Veränderungen in der Experimentalgruppe, die in der Kontrollgruppe nicht zu beobachten waren.

Aus diesen Ergebnissen schließen die Autoren, dass die Behandlung mit Chinakräutern der konventionellen Therapie überlegen sei.

\section{Kommentar}

Dies ist ein Paradebeispiel für die besorgniserregende Qualität vieler Studien aus diesem Bereich. Auf den ersten Blick liest sie sich wie eine Untersuchung, die die Ansprüche der EBM in etwa erfüllt. Auf den zweiten Blick tauchen dann erhebliche Bedenken auf. So fehlt z. B. trotz großer "Drop-out"-Zahlen eine "Intention to treat"-Analyse. Ferner sind die verschiedenen Interventionen nur sehr vage beschrieben, sodass eine Replikation der Studie unmöglich wäre. Am schwersten wiegt vielleicht, dass die Schlussfolgerungen letztlich nicht auf den erstellten Daten beruhen: Die Studie hat kein klar positives, sondern eher ein negatives Ergebnis! Derzeit werden immer mehr derartige Studien in Englisch publiziert. Somit sind sie nunmehr auch für nicht Chinesisch sprechende Leser zugänglich. Die Gefahr ist hier meines Erachtens, dass eine unkritische Betrachtung dieser Publikationen zu falsch positiven Ergebnissen führt, die letztlich zu einem falschen Bild über die Wirksamkeit der TCM beiträgt. Wir sollten also wachsam bleiben und diese Untersuchungen stets genau unter die Lupe nehmen.

E. ERNST .

\section{- R.-H. ZHAO et al.}

Chinese medicine improves postoperative quality of life in endometriosis patients: a randomized controlled trial. Chin. J. Integr. Med. 2013 (1):15-21. doi: 10.1007/s11655-012-1196-6.

\section{Unspezifischer Rückenschmerz: Akupunktur sticht übliche Therapie}

\section{0\% aller Patienten mit unspezi- fischen Schmerzen im Bereich der Lendenwirbelsäule erholen sich innerhalb von sechs Wochen. Daher ist es durchaus möglich, dass hier die guten Ergebnisse der Akupunk- tur auf den Spontanverlauf der Be- schwerden zurückzuführen sind.}

- Die Studie wurde in Spanien durchgeführt. 275 Patienten wurden innerhalb von 14 Tagen nach dem Auftreten von unspezifischen Schmerzen im Bereich der Lendenwirbelsäule in vier Therapiegruppen eingeteilt: 1 . konventionelle Behandlung mit Bettruhe und/ oder nicht steroidalen Antirheumatika, 2. chinesische Akupunktur mit fünf Sitzungen über zwei Wochen, 3. Scheinakupunktur, bei der die Akupunkturnadeln am Rücken jenseits von chinesischen Akupunkturpunkten platziert wurden, 4. Placeboakupunktur, bei der nur lokaler Druck am Rücken ausgeübt

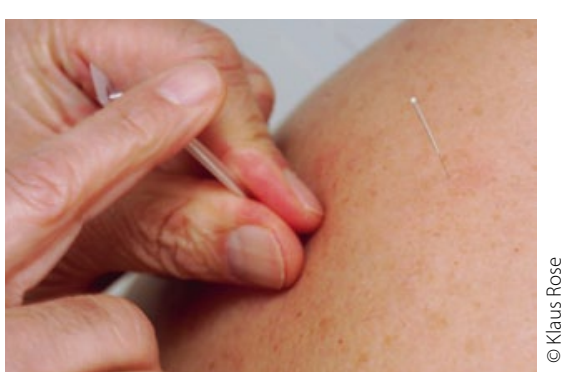

Akupunktur: Nur Placebo oder echter biologischer Effekt?

wurde, der Patient aber nicht wusste, dass keine Nadeln platziert worden waren.

Eine klinisch relevante Verbesserung gaben $73,5 \%$ der Patienten an mit der chinesischen Akupunktur, 75\% mit der Scheinakupunktur, 65\% mit der Placeboakupunktur und $44,3 \%$ in der Kontrollgruppe. Der Unterschied zwischen den drei Akupunkturgruppen und der Kontrollgruppe war statistisch signifikant.

\section{Kommenta}

Diese gut durchgeführte Studie aus Spanien reiht sich ein in eine ganze Reihe von Akupunkturstudien, die in den letzten Jahren durchgeführt wurden. Die meisten dieser Studien zeigten eine Überlegenheit der Akupunktur gegenüber einer konservativen Therapie. Die meisten Studien erbrachten allerdings auch keinen Unterschied zwischen einer echten chinesischen Akupunktur, einer Scheinakupunktur und in einigen Studien gegenüber einer Placeboakupunktur. Dies legt sehr nahe, dass es sich bei dem Behandlungseffekt bei der Akupunktur um einen ausgeprägten Placeboeffekt handelt und nicht um den unterstellten biologischen Effekt der Akupunktur selbst. H.-C. DIENER -

- J. Vas et al.

Acupuncture in patients with acute low back pain: A multicentre randomised controlled clinical trial. Pain 153 (2012) 1883-1889 